\section{Naturheilverfahren in der Dermatologie}

D as Werk „Naturheilverfahren bei Hauterkrankungen" stammt von Matthias Augustin, einem ausgewiesenen Spezialisten der Pharmakoökonomie und Experten auf dem Gebiet der Evidence-based Medicine. Umso erstaunlicher ist es, dass sich Augustin auch mit Naturheilverfahren in der Dermatologie befasst. Für die Verfahren gibt es, wie der Autor selbst im Vorwort einräumt, nur selten fundierte Literaturangaben, wie sie von der Evidenz-basierten Medizin gefordert werden.

Das schön aufgemachte Werk gliedert sich in fünf Abschnitte. In einem einleitenden Kapitel werden Grundlagen der Naturheilverfahren erklärt. Der zweite Abschnitt befasst sich mit dermatologischer Diagnostik und könnte sich ebenso in einem Lehrbuch der wissenschaftlichen Medizin finden. Im dritten Abschnitt werden die dermatologischen Erkrankungen und ihre naturheilkundliche Therapie abgehandelt. Im vierten Abschnitt erfolgt eine ausführliche Darstellung der naturheilkundlichen Verfahren, gegliedert in
Akupunktur, anthroposophische Medizin, ausleitende Verfahren, Bach-Blütentherapie und so weiter. Zur Abrundung trägt der fünfte Abschnitt bei, der „Suchen \& Finden“ genannt wurde. Hier gibt es unter anderem eine Liste der Heilpflanzen mit dermatologischen Indikationen sowie eine Liste mit pflanzlichen Externa einschließlich Handelsnamen und Indikationen.

Als nicht naturheilkundlich geschulter Ärztin fehlt der Rezensentin das rechte Verständnis für manche Verfahren wie Eigenbluttherapie oder Gabe von Enzympräparaten. Eigentümlich erscheint einiges, zum Beispiel, dass in der Heilpflanzenliste die Ringelblume zur Behandlung von Ekzemen, Entzündungen im Mund- und Rachenraum und kleineren Wunden empfohlen wird. Nebenwirkungen seien keine bekannt. Aus eigener klinischer Erfahrung ist die Ringelblume als nicht seltener Auslöser von allergischen Kontaktekzemen durchaus bekannt. Lobenswert ist, dass auch die Grenzen der $\mathrm{Na}$ turheilkunde aufgezeigt werden. Bei- spielsweise wird bei der Onychomykose festgestellt, dass hier keine sinnvolle naturheilkundliche Therapie möglich ist. Es ist hier nicht der Platz, um Sinn oder Unsinn von Naturheilverfahren in der Dermatologie zu diskutieren. Augustin versucht in dem vorliegenden Werk den Spagat, zwei oder gar mehrere unterschiedliche medizinische Denkansätze zusammenzuzwingen, die nicht zusammenpassen wollen.

Alles in allem handelt es sich bei „Naturheilverfahren bei Hauterkrankungen" um ein liebevoll aufgemachtes Werk, an dem Freunde der Naturheilverfahren sicherlich ihre Freude haben werden. Für andere kann es als Informationsquelle dienen.

Dr. Andrea Niedermeier, München

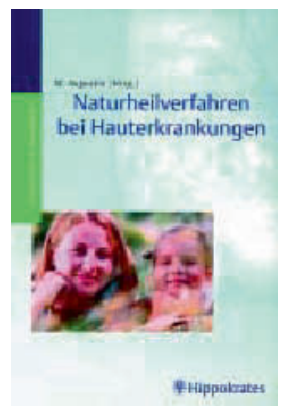

Augustin $M$

(Hrsg.).

Naturheilverfah-

ren bei Haut-

erkrankungen.

Stuttgart:

Hippokrates Ver-

lag, 2002.

$64,95 €$.

ISBN 3-77731-156-1

\title{
Neue Heimat im Netz
}

The homas Kleinoeder und Kurt Wiedenhoff haben ein klares Credo: „Vor wenigen Jahren war der Praxisinhaber mit eigener Homepage ein Exot. Bald wird jeder ein Exot sein, der keine eigene Homepage hat." Wer diesem Satz Glauben schenkt und noch nicht mit einer eigenen Website im Internet vertreten ist, dem sei das neue Buch der beiden - Arzt am medizinischen Rechenzentrum der Universität Göttingen der eine, Marketing-Experte der andere - ans Herz gelegt.

Als ersten Schritt auf dem Weg ins Netz empfiehlt das 116 Seiten starke Werk eine Zielgruppenanalyse: Soll es nur ein virtuelles Praxisschild sein? Sollen Stammpatienten fester an die Praxis gebunden werden oder neue Patienten angesprochen werden? Auf sage und schreibe zwölf Seiten geht es dann um die Wahl der richtigen Internet-Adresse - ein Punkt, den nach Meinung der Autoren gerade Ärzte häufig sträflich vernachlässigen. Ausführlich werden anschließend die Bestimmungen des Teledienst- und des Heilmittelwerbegesetzes erläutert. Auch Internet-Adressen dürfen übrigens keine unzulässige Werbung enthalten: www.weltbesterallergologe.de ist als Domain-Name also tabu.

Erst im letzten Drittel des Büchleins geht es dann richtig zur Sache: Der Leser erfährt mehr über das richtige „Look and Feel“ einer Website, vorbildliche Verlinkung, strukturierte Sitemaps und die Platzierung in Suchmaschinen. Technisch Versierte finden sich im Kapitel „Es geht auch ohne Agentur" wieder. Kommen dabei externe Dienstleister ins Spiel, überzeugen die Autoren durch Praxisnähe: Realistische Kostenabschätzungen in Euro und Cent verhindern, dass der Arzt von unseriösen Providern oder WebdesignAgenturen über den Tisch gezogen wird.

Einziger Wermutstropfen: Layout und vor allem die ohnehin wenigen Abbildungen sind schlicht grau in grau gehalten -- aber hier bleiben die Autoren offenbar dem Bauhausprinzip treu, das sie auch für die Gestaltung von Internet-Seiten empfehlen: „Form folgt Funktion."

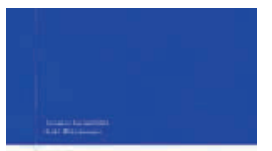

Kleinoeder T, Wiedenhoff K.

Die Homepage für die Arztpraxis

Die Homepage für die Arztpraxis. München: Urban \& Vogel, 2002.

$16,95 €$.

ISBN 3-89935-184-3 